\title{
Notes on Japanese Rubi.
}

(Continued from. No. 178. p. 159).

by

\section{J. Matsumura.}

Rubus palmatus, Thunb. Fl. Jap. p. 217 ; Ic. Pl. Jap. Dec. IV. t. 6 ; Sieb. et Zucc. Fl. Jap. Fam. Nat. I. p. 126 ; Miq. Prol. p. 223 ; A. Gray, Bot. Jap. p. 387, pro parte; Maxim. in Mel. Biol. VIII. p. 384 ; Fr. et Sav. Enum. Pl. Jap. I. p. 126 ; Hemsl. in Jonrn. Linn. Soc. Bot. XXIII, p. 234 ; Palib. Consp. Fl. Kor. p. 78 ; Bot. Mag. (1901), t. 7801. forma coptophylla, O. Kze, Method, p. 95. R. coptophyllus, A. Gray, Perry's Exped. p. 311.

Hab. in Japonia media: prov. Shimotsuke, ad Nikkō (ipse); prov. Musashi, circa urbem Tokyō (ipse); prov. Sagami, tractu Hakone (R. Yatabe); prov. Izumi, ad Nanakoshi-tooge (H. Matsuda); prov. Tamba, ad Kutsukake (S. Oka); prov. Suruga, ins. Awashima (S. Ökulo et ipse);

forma palmata, O. Kze, 1. c.

Hab. in Japonia temperata: prov. J/zu, ins. Ōshima, ad Okata (S. Ōkubo); prov. Suoo, monte T'enjinyama (J. Nikai, no. 264), prov. Izumi : monte Katsuragi (H. Matsuda).

Shikoku: prov. Tosa (T. Makino).

Kiushiu: prov. Ōsumi, ad Ushinegoo (K. Watanabe); prov. Hyuga, monte Kirishima (ipse).

forma ribisifolia, foliis rotundioribus, sub-5-lobatis, lobis mediis brevioribus.

Hab. ins. Ōshima, ad Senzu prov. Izu (S. Ōkubo).

var. remotifolia, Fr. et Sav. Enum. Pl. Jap. II. 334. R. similis, O. Kze, l. c. p. 94.

Hab. in Japonia temperata: prov. Sagami, ad Enoshima (ipse).

Rubus Tanakae, O. Kze, l. c. p. 94, 97 ; Honzoo-zufu, XXV, fol. 
18, recto. Nobis ignota. Sec. Honzoo flores magni solitares; petala extus roseo suffusa.

Rubus peltatus, Maxim. in Mel. Biol. VIII. p. 384; Fr. et Sav Enum. Pl. Jap. I. p. 126 ; Diels in Engl. Bot. Jahrb. XXIX. p. 391. Honzoo-zufu, XXV. fol. 16 verso; Soomokuzusetsu, Arb. ined. V. t. 106.

Folia juv. dense tomentosa. Flores subracemosi. Fructus oblongocylindricus $3 \mathrm{~cm}$. longus, $9 \mathrm{~mm}$. latus. Carpella apice villosa; stipulae amplae membranaceae ovato-semiauriculatae.

Hab. in Hondō: prov. Shinano (coll. ignotus).-Shikoku: prov. Iyo, monte Ishizuchi (S. Ōkubo et R. Yatabe).

Rubus spectabilis, Pursh, Fl. North. Am. I. p. 348. t. 16 ; Miq. Prol. p. 222 ; Maxim. in Mel. Biol. VIII. p. 386 ; Fr. et Sav. Enum. Pl. Jap. I. p. 126.

Hab. in Japonia boreali et media, regionibus montanis: prov. Mutsu, monte Iwakisan (T. Iwakawa); prov. Uzen, montibus Gassan et Chōkaisan (R. Yatabe); prov. Etchu, monte Tateyama (ipse); prov. Kaga, monte Hakusan (M. Toyama et ipse).

Rubus caesius, L. ; DC. Prodr. II. p. 558 ; Ledeb. Fl. Ross. II. p. 66. Caulis prostratus glaber, eglandolosus aculeatus, aculeis recurvis; ramuli puberuli. Folia pinnatim trifoliolata; foliola terminalia longe petiolulata, basi subcordata, lateralia subsessilia, elliptica v. ovalia grosse duplicatodentata interdum lobulata, supra glabriuscula subtus puberula; petioli canaliculati; stipulae persistentes oblengolanceolatae pilosae erectae basi petiolo adantae. Flores solitares axillares v. in terminales reccmas dispositi, $2 \frac{1}{2}$ $\mathrm{cm}$. in diametro; bracteae ovatae v. ellipticae incisae. Calyx $1 \frac{1}{2} \mathrm{~cm}$. in diametro; calycis lobi ovato-lanceolati v. elliptici callosoapiculati, extus parce villosi, intus tomentosi, post anthesin reflexi. Petala late elliptica v. rotundata brevissime unguiculata. Stamina glabra; antherae late ellipticae, carpellis longiora. Carpella numerosa ; ovarium glabrum.

An R. fruticosus, subsp. suberectus, Anderson?

Hav. in Japonia media: prov. Mino, ad Kamagayatsu tractus Ikedagōri leg. K. Mori anno 1887.

Rubus pungens, Camb. var. Oldhami, Maxim. in Mel. Biol. VIII. p. 386 ; Fr. et Sav. Enum. Pl. Jap. I. p. 126 ; Forbes et Hemsl. in Journ. Linn. Soc. Bot. XXIII. p. 236 ; Palib. Cons. Fl. Kor. p. 79 ; Focke in 
Engl. Bot. Jahrb. XXIX. p. 401. R. Oldhami, Miq. Prol. p. 222. Honzoo-zufu, XXV. fol. 14 recto (petala alba majora); Soomoku-zusetsu, Arb. ined. V. t. 92.

Petala carnea, ovalia, suberecto-patentia, breviter unguiculata, calyce longiora.

Hab. in Japonia media: prov. Shimotsuke, regionibus montanis tractus Nikkō (ipse).

Corea : Zenradoo (Hanabusa).

Rubus rosifolius, Sm.; DC. Prodr. II. p. 556 ; Hook. Ic. Pl. t. 349 ; Hook. f. Fl. Brit. Ind. II. p. 341 ; Maxim. in Mel. Biol. VIII. p. 387 ; Miq. prol. p. 222 ; Fr. et Sav. Enum. Pl. Jap. I. p. 126 ; Forbes et Hemsl. in Journ. Linn. Soc. Bot. XXIII. p. 237 ; Henry in Asiat. Soc. Jap. XXIV. Suppl. p. 40; Ito et Matsum. Tent. Fl. Lutch. p. 450 ; Focke in Engl. Bot. Jahrb. XXIX. p. 399. Honzoo-zufu, XXV. fol. 15, recto ; Soomokuzusetsu, Arb. ined. V. t. 88.

Hav. in Japonia temperata: prov. Sagani, tractu Hakone (ipse); prov. Kai, ad Sasago-tonge (R. Yatabe); prov. Shinano (R. Yatabe); prov. Suruga, monte Fuji (ipse)-Shikoku: prov. Iyo, monte Ishizuchi (R. Yatabe) -liukiu: ins. Okinawa (G. Yamada); ins. Oshima (T'. Uchiyama)Formosa: tractu Taitoo (K. Miyake).

Rubus Thunbergii, Sieb. et Zucc. Fl. Jap. Fam. Nat. I. p. 126 ; Miq. Prol. p. 222 ; Maxim. in Mel. Biol. VIII. p. 389 ; Fr. et Sav. Enum. Pl. Jap. I. p. 127 ; Forbes et Hemsl. in Journ. Linn. Soc. Bot. XXIII. p. 238 ; Palib. Consp. Fl. Kor. p. 79. R. hispidus, Thunb. Fl. Jap. p. 216. Fasso Itzingo (ritius Hoso-Itzigo) Kaempf. Am. Exot. p. 787 ; Honzoozufu, XXV. fol. 13, verso ; Soomoku-zusetsu, Arb. ined. V. t. 86.

Hab. iu Japonia temperata: prov. Musashi, tractu Chichibu (jpse); circa urbem Tokyō (ipse); prov. Sagami, tractu Hakone (ipse); prov. Izu (S. Ōkubo); prov. Tōtōmi (S. Ōkubo); prov. Suruga (S. Ōkubo et ispe); prov. Suō (J. Nikai, no. 723)-Kiushiu: prov. Chikuzen (K. Nagano, no. 471)-China: Fengwanchan (F. B. Forbes, no. 155 sub R. rosifolio).

Rubus sorbifolius, Maxim. in Mel. Biol. VIII. p. 390 ; Fr. et Sav. Enum. Pl. Jap. I. p. 127 ; Focke in Engl. Jahrb. XXIX. p. 400. Honzoozufu, XXV. fol. 13, recto ; Soomoku-zusetsu, Arb. ined. V. t. 110.

Hab. in Japonia temperata: prov. Ōmi (ipse).

Rubus Taiwanianus. Matsum. sp. nov. 
Caulis erectus bipedalis v. ultra villosus aculeis erectis v. subrecurvis armatus. Folia pinnata, 1-2-juga; foliola terminalia majora elliptica obtusa v. oblonga acuta v. ovata duplicato- dentata, basi cordata v. rotundata v. acuta, utrinque puberula et punctata; stipulae subulatae. Flores $23 \mathrm{~mm}$. in diametro, axillares solitares v. in terminales recemas dispositi; bractae subulatae. Calycis villosi lobi ovato-lanceolati caudato-acuminati, intus tomentosi. Petala alba, orbicularia integra, unguiculata. Stamina numerosa glabra patentia. Carpella numerosa, glabra; receptaculum pilosum. Fructus exsuccus.

A. $R$. Thunbergiano, S. et $Z$. differt pillis glandulosis longis nullisi tantum punctatis, stipulis semper filiformibus subulatis, floribus parvioribus, fructu exsucco.

Nom. Formos.-sinen.: Chih-poh 䓶波 (ex Niinami).

Hab. in Formosa: Kelung (T. Makino et Owatari), Pikoh, Shalyootoo, Taipeh ('I. Makino), (Honda, no. 129), inter Kusshaku et Shintenkw coll. ignotus), Pachina (Niinami, no. 9, B.).

Rubus fraxinifolius, Poir.; Miq. Fl. Ind. Bat. I. p. 376 ; Maxim. in Mel. Biol. VIII. p. 391.

Fructus coccineus.

Hab. in Formosa : Goshoolon (C. Owatari), ins. Botel-Tobago (K. Miyake).

Rubus tagallus, Cham. et Schtdl. in Linnaea II. p. 9; Maxim. in Mel. Biol. VIII. p. 389 ; Forbes et Hemsl. in Journ. Iinn. Soc. Bot. XXIII. p. 237 ; Henry in Trans. Asiat. Soc. Jap. XXIV. Suppl. p. 40, no. 323.

Hab. in Formosa: Taipeh et Tamsui ('T. Makino); - Liukiu: ins. Okinawa (Y. Tashiro et ipse).

Rubus coreanus, Miq. Prol. p. 222 ; Maxim. in Mel. Biol. VIII. p. 391 ; Forbes et Hemsl. in Journ Linn. Soc. Bot. XXIII. p. 230; Palib. Consp. Fl. Kor. p. 77; Focke in Engl. Bot. Jahrb. XXIX. p. 400. R. Tokkura, Sieb. Syn. Pl. Oeconom. p. 65. no. 345 ; Fr. et Sav. Enum. Pl. Jap. I. p. 128. Honzoo-zufu, XXV. fol. 11, verso ; Soomoku-zusetsu, Arb. ined. V. t. 90.

Folia 1-6-juga, utrinque glabra. Petala carnea erecta obovatosubrotundata margine denticulata.

Hab. in Japonia : circa urbem Tokyō (ipse). Secumdum Honzoo ex Chekiang Chidae advecta. 
Rubus triphyllus, 'Thunb. Fl. Jap. p. 215; Focke in Engl. et Prantl, Nat. Pflanzenf. III. Teil. 2. Ab. a. p. 30; Focke in Engl. Bot. Jahrb. XXIX. p. 397. R. parvifolius, L.; Miq. Prol. p. 222 ; Sieb. et Zucc. Fl. Jap. Farn. Nat. I. 126 ; Maxim. in Mel. Biol. VIII. p. 392 ; Fr. et Sav. Enum. Pl. Jap. I. p. 127; Forbes et Hemsl. in Journ. Linn. Soc. Bot. XXIII. p. 235; Henry in Trans. Asiat. Soc. Jap. XXIV. Suppl. p. 40, no. 320 ; Palib. Consp. Fl. Kor. p. 79 ; Ito et Matsum. Tent. Fl. Lutch. p. 451, R. purpureus, Bunge, Enum. Pl. Chin. Bor. p. 24. R. clinnensis, Thunb. Dissert. de Rubo. p. 8, cum fig. Honzoo-zufu, XXV. fol. 10, recto; Soomoku-zusetsu, Arb. ined. V. t. 94.

Hab. per totam Japoniam: in Yezo: ad Sapporo (K. Miyabe et Y. Tokubuchi), ad Otoibokke (K. Miyabe, fructu luteo), ad Sarachibuto (K. Miyabe), loco non indicato (Boehmer).-Hondō: prov. Mutsu, ad Tsugaru (T. Iwakawa); prov. Uzen, monte Chōkaisan (R. Yatabe); prov. Shimotsuke, tractu Nikkō (R. Yatabe); prov. Sagami, monte Komagatake (R. Yatabe); prov. Izu, ins. Miyake (S. Ōkubo), Konsuitoge ejusdem. prov. (S. Ōkubo); prov. Hitachi (ipse); prov. Shinano (D. Takashima), monte Togakushi, ejusdem prov. (ipse); prov. Kii (Matsubara); prov. Izumi, ad Sakai (H. Matsuda); prov. Yamato, monte Kasuga (ipse).-Shikoku: prov. Awa (R. Yatabe).Kiushiu : prov. Hizen, ad Nagasaki (T. Uchiyama); prov. Chikuzen (K. Nagano), prov. Hyuga (ispe).-Liukiu: ins. Okinawa (Y. Tashiro).-Formosa : Tamsui (T. Makino).-Corea : Ko-ompo (Y. Hanabusa), Keijō ('T. Uchiyama). _China: Lyautoo, ad Taikosan (K. Jimbo); Hanchow (C. Owatari); Fengwanchan (F. B. Forbes, no. 154).

Rubus phoenicolasius, Maxim. in Mel. Biol. VIII. p. 393; Fr. et Sav. Enum. Pl. Jap. I. p. 127 ; Bot. Mag. t. 6479 ; Forbes et Hemsl. in Journ. Linn. Soc. Bot. XXIII. p. 235 ; Focke in Engl. Bot. Jahrb. XXIX. p. 400. R. occidentalis, Thunb. Fl. Jap. p. 216. Honzoo-zufu, XXV. fol. 11, recto; Soomokul-zusetsu, Arb. ined. V. t. 96.

Hab. per totam Japoniam: Yezo: loco non indicato (Boehmer).Hondō: prov. Iwashiro, ad Aizu (ipse); prov. Kaga, monte Hakusan (ipse) ; prov. Shinano, monte Ontake (R. Yatabe), ad Usuitooge, ejusdem prov. (ipse); prov. Shimotsuke, monte Nikkō (K. Sawada); prov. Sagami, ad Hakone (R. Yatabe); prov. Izumi, monte Katsuragisan (H. Matsuda); prov. Kii, monte Kōya (ipse); Tochinokidani (S. Ikeno)-Kiushiu : prov. Buzen, monte Iwatake (ipse); prov. Chikuzen (K. Nagano, no. 629). 
Rubus occidentalis, L. var. japonicus, Miyabe, Fl. Kur. Is. p. 229. R. niveus. Herb. Henry no. 56230. IR. idaeus, var. exsucca, Fr. et Sav. Enum. Pl. Jap. II. p. 334.

Carpella apice pilosa. Fructus initio purpureus, dein niger.

Hab. in Yezo: ad Sapporo (K. Miyabe et ipse), ad Samani-Sandō, Rusuri, Shari-sandō (K. Miyabe).-Hondō: prov. Shimotsuke, montibus Nikkō (ipse); prov. Shinano, monte Komagatake (R. Yatabe), ad Usuitōge (ipse).-China centrali: prov. Hupeh (A. Henry).

Rubus idaeus, L. var. nipponicus, Focke in Abh. Naturw. Ver. Bremen, XIII. p. 471 ; Palib. Consp. Fl. Kor. p. 78.

Pedicelli tomentosi, parce aculeati. Fructus ruber.

Hab. in Hondō: prov. Iwashiro, monte Bandaisan (ipse); prov. Shimotsuke, montibus Nikkō (ipse); prov. Shinano, ad Tamatsukuri (M. Miyoshi); ad Shimizutooge, inter prov. Echigo et Koozuke (T. Makino).

var. strigosus, Maxim. in Mel. Biol. VIII. p. 394 ; Fr. et Sav. Enum. Pl. Jap. I. p. 128.

Hab. in Yezo: loco non indicato (K. Miyabe, fl.), monte Moiwa (ipse, fr.), ad Joozankei (ipse, fr.) loco non indicato (Boehmer, fl. et fr.).-Hondō: prov. Shimotsuke, montibus Nikkō (ipse, fl.).

Pedicelli aculeis pilis glandulosisque dense vestiti.

Petala alba, spatulata subacuta 5-6 $\mathrm{mm}$. longa, 2-3 mm. lata, crecta, decidua. Stamina alba; erecta. 